\title{
INDEX OF VOLUME XXVIII
}

Adams, E. P. See Reviews, under Rougier.

Allen, E. S. See Reviews, under Sommerville.

Bateman, H. See Reviews, under Robb.

BeLL, E. T. Extensions of Dirichlet Multiplication and Dedekind Inversion, 111.

- A Revision of the Bernoullian and Eulerian Functions, 443.

Bernstein, B. A. Reports of Meetings of the San Francisco Section: October Meeting, 94; April Meeting, 281.

- The Complete Existential Theory of Hurwitz' Postulates for Abelian Groups and Fields, 397.

Birkнoff, G. D. See Reviews, under Angerbach, Brouwer, Carmichael, Einstein, Lorentz, Weyl, Whitehead.

Burss, G. A. A Letter from the President, 16.

Bouton, C. L. See Osgood, W. F.

Brown, E. W. See Reviews, under Annuaire.

Cajori, F. See Reviews, under Ampère, Carnot, Clairaut, Laplace, Lavoisier.

CAMP, B. H. A New Generalization of Tchebycheff's Statistical Inequality, 427.

Carmichael, R. D. Note on Euler's $\varphi$-Function, 109.

Algebraic Guides to Transcendental Problems, 179.

See Reviews, under Halphen.

Chase, G. H. See Osgood, W. F.

Chittenden, E. W. Note on the Division of a Plane by a Point Set, 310.

Coble, A. B. Cremona Transformations and Applications to Algebra, Geometry, and Modular Functions, 329.

Cole, F. N. Kirkmann Parades, 435.

Coolidge, J. L. On the Existence of Curves with Assigned Singularities, 451.

See OsGood, W. F.

Cowley, E. B. See Reviews, under Loria.

Crathorne, A. R. See Reviews, under Lipka, Loewy.

Davis, H. T. Relating to the Proof of an Existence Theorem for a certain Type of Boundary Value Problem, 390.

Dickson, L. E. Impossibility of Restoring Unique Factorization in a Hypercomplex Arithmetic, 438.

Dowling, L. W. See Reviews, under Adams, Bally, Deetz.

Dresden, A. A Report on the Scientific Work of the Chicago Section, 1897-1922, 303.

— Eliakim Hastings Moore Fund, 307.

See Reviews, under Knopp, Pascal, Vivanti.

Eisenhart, L. P. Condition that a Tensor be the Curl of a Vector, 425.

Емсн, A. Kinematics in a Complex Plane and some Geometric Applications, 251.

—_ See Reviews, under Emanaud, Halsted, Scheffers. 
Ettlinger, H. J. See Reviews, under Bieberbach, Burkhardt.

Freld, P. See Reviews, under Burali-Forti, Burgatti, Love.

Ford, W. B. On Kakeya's Minimum Area Problem, 45.

Frankuin, P. Two Theorems on Multiple Integrals, 433.

Gillespie, D. C. A Property of Continuity, 245.

Graustein, W. C. See Reviews, under Bouligand.

HART, W. L. Summable Infinite Determinants, 171.

Hildebrandt, T. H. Convergence of Sequences of Linear Operations, 53.

HiLle, E. Convex Distribution of the Zeros of Sturm-Liouville Functions, 261, 462 .

Hitchсоск, F. L. See Reviews, under Naess.

Hurwitz, W. A. Report on Topics in the Theory of Divergent Series, 17.

JACKson, D. Note on an Irregular Expansion Problem, 37.

— See Reviews, under Hahn, Vallée-Poussin.

KellogG, O. D. A Property of certain Functions whose Sturmian Developments do not Terminate, 388. See Reviews, under Rothe.

Lefschetz, S. See Reviews, under Severi.

Lehmer, D. N. See Reviews, under Poletti.

McClenon, R. B. See Reviews, under Heath.

MacMillan, W. D. The Moment of Inertia in the Problem of $N$ Bodies, 165.

Mathewson, L. C. See Reviews, under Baumgartner, Poirée.

Miller, G. A. The Simple Group of Order 2520, 98.

- Substitutions Commutative with every Substitution of an Intransitive Group, 168.

Milne, W. E. A Theorem of Oscillation, 102.

Moore, C. L. E. See Reviews, under Lotze.

Moore, C. N. See Reviews, under Carslaw, Hobson, Pringsheim, Wangerin.

Morgan, F. M. See Reviews, under Hilton.

Osgood, W. F., Coolidge, J. L., and Chase, G. H. Charles Leonard Bouton, 123.

Phillips, H. B. See Reviews, under Reiche.

Pitcher, A. D. See Reviews, under Encyklopädie.

Ponter, M. B. A Two-Way Infinite Series for Lebesgue Integrals, 105.

Reynolds, C. N. See Reviews, under Fabre, Kohler.

Richardson, R. G. D. Reports of Meetings of the American Mathematical Society: Twenty-Eighth Summer Meeting, 1; October Meeting, 89; Twenty-Eighth Annual Meeting, 148; February Meeting, 233; April Meeting, 285; Twenty-Ninth Summer Meeting, 377.

Shaw, J. B. See Reviews, under Appell, Bhattacharyya, Isenkrahe.

Sisam, C. H. See Reviews, under Berzolari.

Smith, D. E. See Reviews, under Bibliotheca, Bryant, Lefebvre.

SNyder, V. See Reviews, under Klein, Schmid.

Southald, J. P. C. See Reviews, under Huygens.

Stouffer, E. B. Report of the Fourteenth Regular Meeting of the Southwestern Section, 145. 
- See Reviews, under Kommerell.

Van der Vries, J. N. See Reviews, under Schuh.

VANDiver, H. S. Note on some Results concerning Fermat's Last Theorem, 258.

— On Kummer's Memoir of 1857 concerning Fermat's Last Theorem, 400.

Van Vleck, E. B. The Bôcher Memorial Prize, 42.

Wahlin, G. E. See Reviews, under Bianchi, Landau, Mordell.

WALSH, J. L. A Generalization of Normal Congruences of Circles, 456.

Williams, K. P. Note concerning the Roots of an Equation, 394.

Wirson, E. B. See Reviews, under Jeans, Schaefer.

Young, J. W. See Reviews, under Benny, Bisacre, Bolton, Heffter.

Zeldin, S. D. Note on Steady Fluid Motion, 313.

\section{Reviews}

Adams, O. S. Latitude Developments connected with Geodesy and Cartography, L. W. Dowling, 473. See DeEtz, C. H.

Ampère, A. M. Mémoires sur l'Eléctromagnétisme et l'Electrodynamique, F. CAJORI, 417.

Angerbach, A. Das Relativitätsprinzip, G. D. BıккноғF, 215.

Annuaire du Bureau des Longitudes pour 1921, E. W. BRown, 223.

Appell, P. Eléments de la Théorie des Vecteurs et de la Géométrie Analytique, J. B. SHAw, 368.

Bally, E. Géométrie Synthétique des Unicursales de Troisième Classe et de Quatrième Ordre, L. W. Dowling, 133.

Baumgartner, L. Gruppentheorie, L. C. Mathewson, 417.

Benny, L. B. Plane Geometry, J. W. Young, 469.

Berzolari, L. Geometria Analytica (seconda edizione), parte prima, C. H. Sisam, 416.

Bhattacharyya, D. Vector Calculus, J. B. Shaw, 415.

Bianchi, L. Lezioni sulla Teoria dei Numeri algebrici e Principi d'Aritmetica analitica, G. E. WAHLin, 466.

Bibliotheca Chemico-Mathematica, D. E. Sмгтн, 66.

Bieberbach, L. Lehrbuch der Funktionentheorie, I: Elemente der Funktionentheorie, H. J. Ettringer, 467.

— Funktionentheorie, H. J. Ettuinger, 467.

Bisacre, F. F. P. Applied Calculus, J. W. Young, 471.

Boggio, I. See Burali-Forti, C.

Bolton, L. An Introduction to the Theory of Relativity, J. W. YounG, 416.

Bouligand, G. Cours de Géométrie Analytique, W. C. Graustein, 470.

Brouwer, L. E. J. Wiskunde, Waarheid, Werkelijkheid, G. D. BırкноғF, 215.

Bryant, W. W. Kepler, D. E. Sмiтh, 67.

Burali-Forti, C., e Boggio, I. Meccanica Razionale, P. Field, 71.

Burkhardt, H. Funktionentheoretische Vorlesungen, erster Band, erstes und zweites Heft, H. J. Etrlinger, 475.

— See Encyklopädie. 
Burgatti, P. Lezioni di Meccanica Razionale (seconda edizione), P. FIELd, 64.

Carmichael, R. D. The Theory of Relativity (second edition), G. D. BirkнOFF, 215.

Carnot, L. Réflexions sur la Métaphysique du Calcul Infinitésimal, I-II, F. CAJORI, 273.

Carslaw, H. S. Introduction to the Theory of Fourier's Series and Integrals (second edition), C. N. Moore, 266.

Clairaut, A. C. Eléments de Géométrie, I-II, F. CAJori, 133.

Deetz, C. H., and Adams, O. S. Elements of Map Projection, L. W. Dowling, 473.

Einstein, A. Relativity. The Special and the General Theory, translated by R. W. Lawson, G. D. Birkhoff, 215.

- See Lorentz, H. A.

Emanaud, M. Géométrie perspective, A. Емсн, 475.

Encyklopädie der Mathematischen Wissenschaften, volume II, part II. Edited by H. Burkhardt, W. Wirtinger, R. Fricke, and E. Hilb, A. D. Pitcher, 474.

Fabre, L. Les Théories d'Einstein, C. N. Reynolds, 224.

Fricke, R. See Encyklopädie.

Hahn, H. Theorie der Reellen Funktionen, erster Band, D. JACKson, 408.

Halphen, G. H. Euvres, volume III, R. D. Carmichael, 271.

Halsted, G. B. Girolamo Saccheri's Euclides Vindicatus, A. Емсн, 131.

Heath, T. The Copernicus of Antiquity (Aristarchus of Samos), R. B. McClenon, 368.

Heffter, L. Die Grundlagen der Geometrie als Unterbau für die Analytische Geometrie, J. W. Young, 224.

Hilb, E. See Encyklopädie.

Hilton, H. Plane Algebraic Curves, F. M. Morgan, 415.

Hobson, E. W. The Theory of Functions of a Real Variable and the Theory of Fourier's Series (second edition), volume 1, C. N. Moone, 266.

Huygens, C. Euvres complètes, tome 13, J. P. C. Southall, 211.

Isenkrahe, C. Untersuchungen über das Endliche und das Unendliche, erstes, zweites und drittes Heft, J. B. SHaw, 367.

Jeans, J. H. The Mathematical Theory of Electricity and Magnetism (fourth edition), E. B. WiLson, 412.

- Problems of Cosmogony and Stellar Dynamics, E. B. Wilson, 413.

Klein, F. Gesammelte mathematische Abhandlungen, Band 1, V. SNYDER, 125.

Knopp, K. Funktionentheorie (zweite Auflage), erster und zweiter Teil, A. DREsDen, 272.

Kohler, K. M. Das Exzentrizitätsprinzip als Korrelat zur Relativitätstheorie, C. N. Reynolds, 319.

Kommerell, K. See Kommerell, V.

Kommerell, V., und Kommerell, K. Allgemeine Theorie der Raumkurven und Flächen (dritte Auflage), erster und zweiter Band, E. B. SToufFER, 317. 
Landau, E. Einführung in die elementare und analytische Theorie der algebraischen Zahlen und der Ideale, G. E. WaHLin, 466.

Laplace, P. S. Essai philosophique sur les Probabilités, F. Cajori, 417. See Lavoisier, A. L.

Lavoisier, A. L., et Laplace, P. S. Mémoire sur la Chaleur, F. CAJori, 417. Lawson, R. W. See Einstein, A.

Lefebvre, B. Notes d'Histoire des Mathématiques, D. E. Sмгтн, 62.

Lipka, J. Graphical and Mechanical Computation, A. R. Crathorne, 272.

Loewy, A. Mathematik des Geld- und Zahlungsverkehrs, A. R. CraTHORNE, 65.

Löffler, E. See Severi, F.

Lorentz, H. A., Einstein, A., und Minkowski, H. Das Relativitätsprinzip (dritte Auflage), G. D. Birkhoff, 215.

Loria, G. Storia della Geometria Descrittiva dalle Origini sino ai Giorni Nostri, E. B. Cowley, 414.

Lotze, A. Die Grundgleichungen der Mechanik, C. L. E. Moore, 472.

Love, A. E. H. Theoretical Mechanics (third edition), P. FIELd, 366.

Masius, M. See Rougier, L.

Minkowski, H. See Lorentz, H. A.

Mordell, L. J. Three Lectures on Fermat's Last Theorem, G. E. WAHurn, 318.

Naess, A. Zur Theorie der Triaden, F. L. Hiтchсоск, 366.

Pascal, E. Lezioni di Calcolo Infinitesimale, I-II (quarta edizione), III (seconda edizione), A. Dresden, 315.

Poirée, J. Précis d'Arithmétique, L. C. Mathewson, 472.

Poletti, L. Tavole di Numeri Primi entro Limiti Diversi, e Tavole Affini, D. N. LeHMer, 70.

Pringsheim, A. Vorlesungen über Zahlen- und Funktionenlehre, ester Band, dritte Abteilung, C. N. Moore, 463.

Reiche, F. Die Quantentheorie, ihr Ursprung und ihre Entwicklung, H. B. Phillips, 69.

Robb, A. A. The Absolute Relations of Time and Space, H. Bateman, 318.

Rothe, H. Vorlesungen über Höhere Mathematik, O. D. KellogG, 468.

Rougier, L. Philosophy and the New Physics, translated by M. Masius, E. P. ADAMs, 319.

Saccheri, G. See Halsted, G. B.

Schaefer, C. Einführung in die theoretische Physik, Band II, Teil 1, E. B. WILSON, 222.

Scheffers, G. Lehrbuch der Darstellenden Geometrie, erster und zweiter Band, A. Емсн, 130.

Schmid, T. Darstellende Geometrie, zweiter Band, V. SNYder, 68.

Schuh, F. Lessen over de Hoogere Algebra, volume 1, J. N. VAN DER VRIES, 474.

Severi, F. Vorlesungen über algebraische Geometrie. Translated by E. Löffler, S. LEFSCHETz, 365.

Sommerville, D. M. Y. The Elements of Non-Euclidean Geometry (second edition), E. S. Allen, 223. 
Vallée Poussin, C. de la. Leçons sur l'Approximation des Fonctions d'une Variable Réelle, D. JACKson, 59.

Vivanti, G. Lezioni di Analisi Infinitesimale (seconda edizione), A. DresDEN, 315.

Wangerin, A. Theorie des Potentials und der Kugelfunktionen, zweiter Band, C. N. Moore, 465.

Weyl, H. Raum, Zeit, Materie, G. D. Birкноғғ, 215.

Wirtinger, W. See Encyklopädie.

Whitehead, A. N. The Concept of Nature, G. D. Birкнofr, 215.

Index of Volume XXVIII, 493.

New Publications, 83, 139, 228, 278, 325, 373, 421, 479.

Notes, 72, 134, 225, 274, 320, 369, 418, 476 .

Papers Read before the Society and Subsequently Published, Thirty-First Annual List of, 484.

\section{Notes AND OTHER ITEMS}

Academies, Associations, Congresses, and Societies:

American Mathematical Society: Annual Meeting; Annual Meeting (1922), 90, 369; April Meeting, 72, 152; Bôcher Memorial Prize, 42, 378; Bulletin, 150, 274; Chicago Section, December Meeting, 369, April Meeting, 476; Elections of Officers: of the Society, 151; of the Chicago Section, 151; of the San Francisco Section, 94; of the Southwestern Section, 145; February Meeting, 72; Life Memberships, 235; Moore Fund, 135, 286, 378; New Members Admitted, 2, 89, 149, 233, 234, 286, 378; Reciprocity Agreements, 3, 378; Representatives: on Council of American Association for the Advancement of Science, 150; on National Research Council, 75, 235; Southwestern Section Meeting, 369; Statistics, 151; Summer Meeting, 134, 150; Transactions, 72, $234,235,286,476$.

Association for the Advancement of Science: American, 134, 150. Accademia dei Lincei, 418; American Astronomical Union, 135; Les Amis des Nombres, 135; Belgian Academy, 74, 135, 378; Belgian Mathematical Society, 135, 378; British Commissioners of 1851, 320; Franklin Institute, 477; Helmholtz-Gesellschaft, 73; International Astronomical Union, 320; International Research Council, 477; Italian Society of Sciences, 136; Jablonowski Society, 320; LeopoldinischCarolinische Akademie, 418; London Mathematical Society, 135; Mathematical Association of America, 74; National Academy of Sciences, 275, 476; National Council of Mathematics Teachers, 225; National Research Council, 75, 225, 235; Norwegian Mathematical Society, 73; Optical Society of America, 135; Paris Academy of Sciences, 135, 477; Petrograd Physico-Mathematical Society, 369; Royal Astronomical Society, 136; Royal Society of Edinburgh, 320; Royal Society of London, 136, 477; Società Ligustica di Scienze e Lettere, 369; Vienna Academy of Sciences, 74.

Books, Announcement of New, 73, 135, 418.

Doctorates in Mathematics, American, 275.

Journals:

Abhandlungen aus dem Mathematischen Seminar der Hamburgischen Universität, 134; American Journal of Mathematics, 72, 274, 476; American Mathematical Monthly, 134; Annali di Matematica, 
370; Annals of Mathematics, 72, 418, 476; Bollettino di Bibliografia e Storia delle Scienze Matematiche, 274; Bollettino di Matematica, 274; Bulletin of the American Mathematical Society, 150, 274; Christiaan Huygens, 134; Jahrbuch über die Fortschritte der Mathematik, 73; Journal of Mathematics and Physics of the Massachusetts Institute of Technology, 73; Masaryk University Memoirs, 134; Mathematische Annalen, 73; Mathematische Zeitschrift, 73; Publications de l'Institut Mathématique de l'Université de Strasbourg, 476; Revista Matematica din Timisoara, 134; Sphinx-Edipe, 135; Transactions of the American Mathématical Society 72, 234, 235, 286, 476; Warsaw Polytechnic Society Memoirs, 134.

Papers and Communications Presented to the Society, Authors:

Alexander, J. W., 10, 10.

Beatty, S., 161.

Bell, E. T., 96, 97, 97, 97, 282, $283,283$.

Bernstein, B. A., 96, 282.

Birkhoff, G. D., 5, 5, 236, 236.

Blichfeldt, H. F., 284.

Bliss, G. A., 294.

Buck, T., 95.

Burgess, R. W., 154.

Cajori, F., 95, 95, 281, 282, 284.

Camp, B. H., 14.

Camp, C. C., 383.

Campbell, G. A., 94.

Carlson, E., 290, 381.

Carmichael, R. D., 152, 287.

Carpenter, A. F., 283.

Carr, F. E., 294.

Chittenden, E. W., 147, 297.

Clarke, E. H., 383.

Coble, A. B., 286.

Cole, F. N., 380.

Coolidge, J. L., 239.

Copeland, L. P., 291.

Cowling, A. H., 384.

Crum, W. L., 13, 14, 156, 292, 292.

Cummings, L. D., 163.

Curtiss, D. R., 152, 159, 287, 288.

Daus, P. H., 281.

Davis, H. T., 298, 299.

Dickson, L. E., 300, 301.

Dodd, E. L., 9, 158, 384.

Douglas, J., 238.

Dresden, A., 295.

Dunkel, O., 146.

Dushman, S., 152.

Eiesland, J., 4.

Eisenhart, L. P., 3, 154, 238, 379.

Emch, A., 157, 241.

Ettlinger, H. J., 384.

Evans, G. C., 382, 382.

Everett, H. S., 158.

Eversull, B. M., 289.
Fischer, C. A., 7, 241, 386.

Forsyth, C. H., 156.

Franklin, P., 384.

Fry, T. C., 296.

Gilman, R. E., 12.

Glashan, J. S. C., 160, 160.

Glenn, O. E., 12, 163, 292.

Gokhale, V. D., 302.

Graustein, W. C., 242, 381.

Gronwall, T. H., 9, 93, 239, 239.

Gummer, C. F., 12, 160.

Hart, W. L., 301.

Hausle, E. C., 7.

Hazlett, O. C., 164.

Hedrick, E. R., 146.

Hille, E., 159.

Hoar, R. S., 296.

Hodge, F. H., 297.

Hollcroft, T. R., 243.

Huntington, E. V., 5, 155, 239, 240.

Hurwitz, W. A., 156.

Ingold, L., 146.

Ingraham, M. H., 153.

Kasner, E., 4, 92.

Kellogg, O. D., 236, 236, 385.

Kendall, C., 145.

Kline, J. R., 8, 8, 380.

Lane, E. P., 298.

Langer, R. E., 236, 237.

Lefschetz, S., 146, 147, 379.

Lennes, N. J., 299, 300, 380.

Lipka, J., 157.

Lunn, A. C., 3.

McLennan, J. C., 152.

MacNeish, H. F., 158.

Manneback, C., 242.

Miller, G. A., 5, 155, 156, 290, 382.

Moore, C. N:, 11, 288, 380.

Moore, E. H., 161, 296.

Moore, R. L., 9, 91, 92, 293, $293,380$.

Morenus, E. M., 242.

Moritz, R. E., 96.

Morris, F. R., 284. 
Morse, H. C. M., 10.

Murray, F. H., 385, 386.

Musselman, J. R., 13.

Myller, A., 381.

Noble, C. A., 283.

Pfeiffer, G. A., 240.

Phillips, H. B., 152.

Pierpont, J., 3.

Porter, M. B., 15.

Pounder, I. R., 161.

Press, A., 243.

Reilly, J. F., 295.

Rice, L. H., 93.

Rider, P. R., 289.

Ritt, J. F., 90, 91, 241.

Robinson, L. B., 94.

Roever, W. H., 147, 302.

Rowe, J. E., 13.

Schmidt, K., 381.

Schwatt, I. J., 6, 6, 6, 163, 163, 244, 244, 386, 387, 387.

Sharpe, F. R., 379.

Shaw, J. B., 295.
Slaught, H. E., 152.

Snyder, V., 379.

Speiser, A., 294.

Stouffer, E. B., 147, 289.

Synge, J. L., 7.

Tolman, R. C., 152.

Turner, J. S., 297.

Veblen, O., 154.

Walsh, J. L., 12, 93, 164, 241.

Webster, A. G., 11, 11.

Westfall, W. D. A., 146.

Whittemore, J. K., 90.

Wiener, N., 15, 164, 291.

Wilczynski, E. J., 162.

Wilder, C. E., 153, 153.

Wilder, R. L., 299.

Williams, A. R., 96.

Williams, K. P., 298.

Williams, W. L. G., 385.

Wong, B. C., 95.

Woods, R., 294.

Yerkes, R. M., 152.

Zeldin, S. D., 15, 155, 291, 381.

Personal Notes:

Abbe, E., 275; Alderton, N. M., 80, 275, 372; Aley, R. J., 79; Allen, E. S., 80; Almansi, E., 136; Alrich, G. F., 138, 371; Anderson, W. E., 372; Andrade, J., 136; Andreoli, G., 419; Appuhn, W. E. F., 372; Aprile, G., 419; Archibald, R. C., 370; Armellini, 136, 418; Armstrong, B., 80, 275; Atwater, F. A., 372.

Bäcklund, A. V., 478; Bagnera, G., 137; Bailey, H. W., 420; Baire, R., 135, 275; Ball, W. W. R., 320; Ballantine, J. P., 138; Banerji, S. K., 478; Barton, H., 323; Bateman, H., 323; Bauer, E., 277; Baule, B., 76; Beale, F. S., 420; Beatley, R., 138; Beckwith, E. R., 138; Bedard, A. J., 80; Beetle, R. D., 371; Beghin, H., 419; Bell, E. T., 324; Bell, H. A. D., 420; Beman, W. W., 82, 138; Benedict, S. R., 79; Bennett, R., 80; Bernays, P., 370; Bernstein, F., 226; Berry, E. M., 275; Bertini, E., 323; Berwick, W. E. H., 137; Birkhoff, G. D., 225, 225, 226; Blaschke, W., 134; Blincoe, J. W., 324; Bliss, G. A., 225; Blodgett, R., 81, 275; Blumenthal, O., 73; Bôcher, M., 74, 225; Boerckel, B. M., 324; Bohr, N., 136, 136, 477; Bond, J. D., 79, 324; Bouton, C. L., 82, 123; Boutroux, P., 135, 325; Bowie, W., 225; Bramble, C. C., 371; Brand, L., 371; Brandt, H., 226; Branson, J. W., 80; Breuer, S., 77; Briggs, G. B., 420; Brillouin, M., 136; Brinkman, H. W., 80; Brow, E., 420; Brown, B. H., 324; Brown, F. L., 419; Brown, T. A., 225; Brown, T. H., 478; Browne, E. T., 79, 371; Bryan, N. R., 372; Bunn, H. S., 420; Burgatti, P., 370; Burkett, F. J., 371; Burnam, J. E., 420; Burwell, W. R., 323.

Cailler, C., 138; Cajori, F., 75; Calkins, H., 80; Camp, C. C., 80; Campbell, J. E., 135; Campbell, J. W., 77; Campbell, W. W., 320; Cannon, A. J., 75; Cardin, C. J., 80; Carr, F. E., 78; Carroll, I. S., 138; Carter, M. B., 81; Carus, M. H., 74; Chadbourne, M. F., 420; Chambers, G. G., 78; Chandler, E., 78; Chandler, H. W., 277; Chatelet, A., 323; Churchill, R. V., 324; Cisotti, U., 137; Clark, Mrs. T., 80; Coates, W. M., 138; Coble, A. B., 72, 286; Coit, J. B., 277; Colpitts, J. T., 323; Conti, A., 274; Conwell, H. H., 419; Coolidge, J. L., 72; Cotterill, H. J., 138; Cotton, E., 137, 226; Craig, C. C., 324; Cresse, G. H., 79; Crum, W. L., 227; Cummings, H. K., 138, 323. 
Dadourian, H. M., 371; Dalaker, H. H., 79; von Dalwigk, F., 477; Dantscher von Kollesburg, V., 81; Darmois, 137; Darwin, C. G., 78; Daus, P. H., 80, 275, 324; Davis, C. H., 420; Davis, H. J., 77; Davis, J. E., 372; Davis, N. F., 81; Dehn, M., 76; Deltheil, 137; De Lury, A. T., 371, 478; Deming, R. M., 478; Dickson, L. E., 75, 235; Dines, L. L., 137; Dixon, A. L., 135, 227; Doan, C. S., 79; Dobell, H. A., 80; Dodd, E. L., 419; Doetsch, G., 77; Doležal, E., 74; Doll, T., 419 Donati, L., 77; de Donder, T., 135; Donohue, J. N., 371; Dostal, B. F., 138; Drach, J., 136, 226; Dresden, A., 80, 286; Duane, W., 225; Durfee, W. H., 277.

Easterbrooks, R. T., 80; Eddington, A. S., 225, 275; Eddy, H. T., 82; Edington, W. E., 78, 275, 324; Einstein, A., 75, 136, 275, 477; Eisenhart, L. P., 275; Eisenmann, K., 136; Elliott, E. B., 227; Elliott, W. W., 277; Enriques, F., 137; Eshleman, J. D., 80.

Falckenberg, H., 371; Fehr, H., 75; Feyer, E., 419; Fischer, C. A., 478; Fithian, J. H., 324; Foord-Kelcey, W., 138; Foster, M. C., 81, 275; Foster, R. M., 80; Fraenkel, A., 418; Francis, E. C., 478; Franklin, P., 138, 275, 277; Fredholm, E. I., 225; Frost, E. B., 225; Fuhner, H. K., 324; Funk, J. C., 138; Funk, P., 76.

Gaillot, J. A., 81; Gale, A. S., 227; Gale, H. G., 225; Gambier, 323; Gant, A. E., 80; Gérardin, A., 135; Gerrans, H. T., 81; Giraud, G., 137; Glazebrook, R., 75; Godeaux, L., 135; Goodwin, H. M. 73; Gossard, H. C., 323, 419, 478; Grammel, R., 76; Grassmann, H., 76, 325; Greenhill, G., 370; Gubler, E., 138; Gummer, C. F., 478; Gutzmer, A., 225; Gutzmer, M., 418.

Hagen, J. G. 477; Hahn, H., 74, 74, 225; Halsted, G. B., 277; Hammond, E. S., 79; Hanna, U. S., 371; Hardy, G. H., 135, 135, 370, 370; Hardy, J. G., 419; Harper, F. S., 324; Haskins, C. N., 75, 225, 235, 378; Haupt, O., 77; Hausdorff, F., 77; Hayden, C., 420; Hecke, E., 134; Hegeler, E. C., 74; Helmholtz, H. L. F., 73, 135; Hennel, C. B., 371; Heun, K., 74; Hiches, D., 419; Hicks, H. C., 324; Hickson, A. O., 324; Hilbert, D., 73, 369; Hill, L. S., 78, 227; Hill, W. H., 323; Hille, C. E., 80, 420; Hinsch, V. B., 372; Hodge, F. H., 79; Höfler, A., 420; Holbert, K. W., 277; Holgate, T. F., 78; Holler, Z. N., 420; Holman, W. F., 79; Horn, J., 74; Hotopp, L., 419; Howe, A. M., 372; Humbert, G., 77, 135; Humbert, P., 137; Hyde, E., 79.

Ingalls, E., 420; Ingham, A. E., 320.

Jackson, R. L., 420; Jaeger, C. G., 324; Jahnke, E., 227; James, G., 478; Janet, 478; Jeans, J. H., 136, 137, 420, 477; Jeffery, G. B., 227; John, F. W., 324; Johnson, M. M., 420; Johnson, R. P., 138, 371; Johnston, L. S., 371; Jones, J. E., 320; Jordan, C., 81, 320; Joukowski, N., 81 .

Kaluza, T., 418; Kapteyn, J. C., 325; Kasner, E., 135; Keller, E. B., 324; Keller, E. G., 80; Kellogg, O. D., 75; Kempe, A. B., 277; Kendall, C., 138, 324; Kennelly, A. E., 370; Kennon, R. E., 324; Kenyon, A. M., 82; Keyes, F. G., 73; Kiefer, E. C., 323; Kiepert, L., 76; Killam, S. D., 77; Kingston, H. R., 78; Kneser, A., 74, 369; Knox, J., 80; Kohn, G., 227; Kommerell, K., 226; Konantz, E. L., 137; König, H., 477; König, R., 226, 418; Königsberger, L., 227; Kostka, C., 420; Kötter, E., 420; Kruppa, E., 76, 477; Kuschke, C. G. P., 78, 227.

Lamb, R. C., 138; Lambert, C. J., 138; Landau, E., 74, 370; Landis, W. W., 79; Lang, P. S., 137, 370; Langmuir, I., 75; La Paz, L., 80; Larew, G. A., 419; Larmor, J., 136; Lasley, J. W., 371; von Laue, 
M., 77; Lazzarino, O., 77; Leahy, A. H., 478; Lebesgue, H., 77, 320; Lebon, E., 420; Lecointe, G., 477; Leduc, 226; Lees, S., 323; Leith, J. D., 420; Lemon, H. B., 420; Leonard, F. C., 420; Levi Civita, T., 320, 477; Lewis, C. S., 79; Lichtenstein, L., 73, 77; Lie, S., 73; Light, G. H., 324; Lipka, J., 370; Lippmann, G., 81, 137; Lipscombe, W. H., 420; Lock, J. B., 138; Logsdon, M. I., 275, 420; Lombardi, L., 370; Lorentz, H. A., 72, 78, 275; Loria, G., 274, 369, 370; Loring, W. E., 420; Lovitt, W. V., 80; Lubben, R., 420; Lyman, T., 78.

McCain, G., 78; McFarland, E., 420; McKelvey, J. V., 80; McKittrick, B. J., 79; McMahon, J., 325; MacDuffee, C. C., 80; Mackie, E. L., 78; MacQueen, M. L., 81; Marcolongo, R., 136; Markov, A., 420; Marquis, R. H., 324; Marshall, W., 79, 323; Mathews, G. B., 227; Mayer, E. S., 138; Meacham, E. D., 372; Mehmke, R., 477; Melville, C. E., 371; Mendenhall, C. E., 225, 225; Merriam, F. M., 324; Michelson, A. A., 135; Mignosi, G., 419; Miller, E. B., 81; Miller, G. A., 134, 370; Miller, N., 371; Milne, E. A., 275, 323; Mirimanoff, D., 419; Miser, W. L., 372; Mitchell, H. H., 78; Mitchell, U. G., 227; Montel, P., 135, 226; Moore, C. L. E., 73; Moore, E. H., 135, 135, 148, 286, 287; Moore, R. L., 369; Morgan, F. M., 324; Morris, F. R., 79; Morse, H. C. M., 227; Morton, A. B., 372; Moulton, E. J., 79; Muir, T., 225; Müller, J. O., 77; Mullemeister, H., 324; Mundorff, R. M., 324; Murnaghan, F. D., 79; Murray, F. H., 478.

Nalli, P., 77; Nancy, A., 372; Nassau, J. J., 137; Nelson, C. A., 277; Newson, M. W., 137; Nicholson, J. W., 137; Nielsen, J., 136; Niven, C., 478; Noble, C. A., 324; Noether, F., 226; Noether, M., 227, 275; Nörlund, N. E., 477; Northcott, J. A., 277; Nowlan, F. S., 137, 324 .

d'Ocagne, M., 136; Osgood, W. F., 225, 227; Ostrowski, A., 477.

Packer, M. C., 324; Page, L., 227; Painter, B. D., 372; Parks, G., 324; Peirce, B., 78; Pérès, J., 137; Pepper, E. D., 324; Pfeiffer, F., 77, 226; Phipps, C. G., 80; Picard, E., 477; Picone, M., 77; Pincherle, S., 75; Planck, M., 225; Plapp, E. M., 137; Plummer, H. C., 77; Pohlhausen, 226; Prange, G., 77, 226.

Rademacher, H., 419; Radon, J., 74, 134, 226; Rankine, A. O., 135; Reidemeister, K., 370; Reinhardt, K., 77; Reynolds, C. N., 78; Reynolds, J. B., 79; Ricci, G., 136; Richmond, H. W., 135; Rider, P. R., 372; Riebesell, P., 77; Riggenbach-Burkhardt, A., 81; Risley, W. J., 323; Robbins, R. B., 324; Roberts, M. M., 80; Robinson, R. G., 324; Robinson, R. M., 420; Roman, I.,.275; Rosenberger, N. B., 79; Russell, H. N., 477; Rutherford, E., 75.

Sabine, W. C., 78; Salkowski, E., 74; Sampson, R. A., 320; von Sanden, H., 477; Saunders, F. A., 225; Scarborough, J. B., 138; Schick, M., 372; Schlink, W., 77; Schmeidler, W., 226; Schoenflies, A., 76; Schuh, F., 134; Schur, J., 369; Schüssler, R., 225; Schuster, A., 477; Schwarz, H. A., 81, 225; Scorza, G., 77; Scott, C. A., 72, 274, 281; Searle, M., 420; Sears, L., 420; Segre, 370; Serini, R., 419; Severi, F., 137; Shaub, H. C., 277; Sheets, R. A., 419; Sherrington, C. S., 135; Shock, J. H., 80; Siegel, C., 477; Silberstein, L., 135; Silla, L., 77, 323; Simpson, C. G., 371; Sinclair, 371; Skiles, W. V., 372; Slotnick, M. M., 277; Smail, L. L., 324; Smith, D. E., 138, 277; Smith, D. M., 372; Smith, Edwin R., 80; Smith, G. W., 227; Snyder, A. D., 80; Snyder, V., 370; Sommerfeld, A., 322, 478; Soula, 419; Sparrow, C. M., 478; Sperry, M., 324; Spingler, W. M., 80; Stäckel, P., 73; Steed, D. V., 78, 275; Stetson, J. M., 80; Stevens, A. H., 420 ; Stocker, J. E., 79; Stone, R. B., 372; Stormer, C., 477; Stouffer, 
E. B., 227, 371; Stromquist, C. E., 419; Sun, J., 275; Sutton, F. D., 275; Swartzel, K. D., 323; Szàsz, O., 226.

Tappan, A. H., 80; Tedone, O., 136, 325; Thaer, A. W. A., 81; Thomae, J. K., 81; Thomas, J. M., 324; Thomson, G. P., 478; Thomson, J. J., 75, 477; Thue, A., 420; Tisdale, W. E., 225; Tolman, R. C., 323; Tonelli, L., 323, 370; Touton, F. C., 138, 372; Trawich, G. T., 420; Tripp, M. O., 78; Trowbridge, A., 225, 225; Turnbull, H. W., 137; Turner, J. S., 80; Turrière, E., 137.

Uhler, H. S., 372; Underhill, A. L., 277; Usai, G., 419.

Vallée Poussin, C. J. de la, 136; Vance, E. H., 80; Vass, J. D., 420; Volterra, V., 477.

Wagner, W. J., 324; Wait, A. H., 324; Walker, E., 419; Walker, G. T., 370; Walsh, J. L., 80; Walton, T. O., 79; Wangerin, A., 225, 418; Wapple, A. R., 81; Ward, L.E., 80; Watson, G. N., 135; Wavre, R., 136, 419; Webster, A. G., 320; Wedderburn, J. H. M., 371; Wells, M. E., 372; Wentworth, G., 138; Western, A. E., 135; Whetsell, H. W., 272; White, F. L., 420; Whitehead, A. N., 72, 75, 274, 320, 370; Whitford, D. E., 80; Whittaker, E. T., 370; Widder, D. V., 277; Wiener, C., 324; Wilder, C. E., 371; Wilder, R. L., 80; Wilkins, P. D., 420; Williams, W. L. G., 227; Willis, A. R., 420; Willis, R., 81; Wilson, D. C., 135; Wilson, E. B., 227; Wilson, W. A., 79; Wilson, W. H., 419; Winters, F. W., 137; Wolfe, C., 323; Wood, F. E., 275, 371; Woods, F. S., 73; Woods, R., 419; Wong, B. C., 324; Wyant, K., 80.

Yeaton, C. H., 78; Young, A. E., 372; Young, A. L., 80; Young, W. H., 135.

Ziwet, A., 276; Zondadari, 137.

Prizes and Medals:

Abbe, 275; Accademia dei Lincei, 418; Belgian Academy, 74, 135; Bôcher Memorial, 42, 378; Cambridge University: Alan Bodey, 136; Rayleigh, 225; Smith, 275; Franklin Institute, 477; Italian Society of Sciences, 136; Jablonowski Society, 320; Leopoldinisch-Carolinische Akademie: Cothenius, Gutzmer, 418; National Academy of Sciences, 476; Nobel, 477; Paris Academy: Bordin, Francœur, Petit d'Ormoy, Saintour, 135; Janssen, Lalande, 477; Royal Astronomical Society, 136; Royal Society of Edinburgh: Keith, James Scott, 320; Royal Society of London: Copley, Hughes, 136; Sylvester, 477; Vienna Academy, 74.

Universities and Technical Schools:

Bologna, 225;

Bryn Mawr, 72, 274;

California Institute, 78 ;

Cambridge, 136, 225, 275, 320;

Chicago, 136, 320;

Columbia, 276;

Cornell, 276;

Harvard, 73, 75, 276;
Illinois, 321;

Johns Hopkins, 321;

Massachusetts Institute, 73, 76, 321;

Michigan, 276, 321;

Pennsylvania, 76, 322;

Rice Institute, 76;

Wisconsin, 72, 322;

Yale, 322 . 